\title{
Use of a digital phantom developed by QIBA for harmonizing SUVs obtained from the state-of-the-art SPECT/CT systems: a multicenter study
}

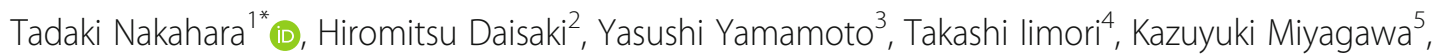
Tomoya Okamoto ${ }^{6}$, Yoshiki Owaki ${ }^{7}, 1$, Nobuhiro Yada $^{3}$, Koichi Sawada $^{4}$, Ryotaro Tokorodani ${ }^{5}$ and Masahiro Jinzaki ${ }^{1}$

\begin{abstract}
Background: Although quantitative analysis using standardized uptake value (SUV) becomes realistic in clinical single-photon emission computed tomography/computed tomography (SPECT/CT) imaging, reconstruction parameter settings can deliver different quantitative results among different SPECT/CT systems. This study aims to propose a use of the digital reference object (DRO), which is a National Electrical Manufacturers Association (NEMA) phantom-like object developed by the Quantitative Imaging Biomarker Alliance (QIBA) fluorodeoxyglucose-positron emission tomography technical committee, for the purpose of harmonizing SUVs in Tc-99m SPECT/CT imaging.

Methods: The NEMA body phantom with determined Tc-99m concentration was scanned with the four state-of-theart SPECT/CT systems. SPECT data were reconstructed using different numbers of the product of subset and iteration numbers (SI) and the width of 3D Gaussian filter (3DGF). The mean ( $\left.S U V_{\text {mean }}\right)$, maximal ( $\left.S U V_{\text {max }}\right)$, and peak (SUV peak $)$ SUVs for six hot spheres $(10,13,17,22,28$, and $37 \mathrm{~mm}$ ) were measured after converting SPECT count into SUV using Becquerel calibration factor. DRO smoothed by 3DGF with a FWHM of $17 \mathrm{~mm}\left(\mathrm{DRO}_{17} \mathrm{~mm}\right)$ was generated, and the corresponding SUVs were measured. The reconstruction condition to yield the lowest root mean square error (RMSE) of $\mathrm{SUV}_{\text {means }}$ for all the spheres between $\mathrm{DRO}_{17} \mathrm{~mm}$ and actual phantom images was determined as the harmonized condition for each SPECT/CT scanner. Then, inter-scanner variability in all quantitative metrics was measured before (i.e., according to the manufacturers' recommendation or the policies of their own departments) and after harmonization.
\end{abstract}

Results: RMSE was lowest in the following reconstruction conditions: S/ of 100 and 3DGF of $13 \mathrm{~mm}$ for Brightview XCT, SI of 160 and 3DGF of 3 pixels for Discovery NM/CT, S/ of 60 and 3DGF of 2 pixels for Infinia, and S/ of 140 and 3DGF of $15 \mathrm{~mm}$ for Symbia. In pre-harmonized conditions, coefficient of variations (COVs) among the SPECT/CT systems were greater than $10 \%$ for all quantitative metrics in three of the spheres, SUV max and SUV mean, in one of the spheres. In contrast, all metrics except SUV max in the 17-mm sphere yielded less than $10 \%$ of COVs after harmonization.

Conclusions: Our proposed method clearly reduced inter-scanner variability in SUVS. A digital phantom developed by QIBA would be useful for harmonizing SUVs in multicenter trials using SPECT/CT.

Keywords: SPECT/CT, Harmonization, SUV, Multicenter study

\footnotetext{
* Correspondence: nakahara@rad.med.keio.ac.jp

'Department of Radiology, Keio University School of Medicine, 35

Shinanomachi, Shinjuku-ku, Tokyo 160-8582, Japan

Full list of author information is available at the end of the article
} 


\section{Background}

Although physical quality of single-photon emission computed tomography/computed tomography (SPECT/CT) images such as image resolution and noise is worse than that of PET/CT images, recent studies suggested the possibility for the clinical application of quantitative SPECT/ CT [1-3]. In 2010, Zeintl et al. reported that the advanced SPECT/CT technology facilitated quantitative Tc-99m SPECT imaging with excellent accuracy in both the phantom (error $<3.6 \%$ ) and patient studies (error $<1.1 \%$ ) [3]. In 2012, Seret et al. investigated the performance of the four state-of-the-art SPECT/CT systems (Philips Brightview XCT, General Electric Discovery NM/CT 670 and Infinia Hawkeye 4, and Siemens Symbia T6) in quantitative assessment using three-dimensional iterative reconstruction (3D-OSEM) with attenuation and scatter corrections and resolution recovery [1]. Quantitative errors of the four SPECT/CT systems were less than $10 \%$ if the targets were several times larger than the spatial resolution of these SPECT devices. In the same year, Hughes et al. also conducted a phantom study in order to compare the images obtained with three different SPECT/ CT systems [2]. Interestingly, their study showed no significant differences in image quality when using their own algorithm, whereas image quality was different between images reconstructed with the vendors' reconstruction software. These results seem to raise a problem with regard to the standardization of SPECT/CT quantitation among different nuclear medicine institutions.

At present, common parameters used for quantitation in clinical SPECT/CT are the maximal standardized uptake value $\left(\mathrm{SUV}_{\max }\right)[4,5]$ and peak SUV $\left(\mathrm{SUV}_{\text {peak }}\right)$ [6]. SUV is the ratio of the radioactivity concentration in a voxel of the target to the average radioactivity concentration in the body, and SUV ${ }_{\max }$ is the highest SUV within a volume of interest (VOI). Although $\mathrm{SUV}_{\max }$ is preferably used in clinical PET imaging because it is not affected by ROI settings, optimization of reconstruction parameter settings is important to harmonize quantitative metrics among different PET cameras [7]. Since SUV is susceptible to spatial resolution and image noise, reconstruction conditions should be properly adjusted for each camera to provide reliable and robust SUVs in terms of the harmonization of SPECT/CT quantitation. In other words, harmonization-specific imaging protocol is crucial for clinical multicenter trials using quantitative SPECT/ $\mathrm{CT}$. This trend has been preceded by fluorodeoxyglucosepositron emission tomography/computed tomography (FDG-PET/CT) for multicenter trials [7, 8].

Recently, the Quantitative Imaging Biomarker Alliance (QIBA) FDG-PET technical committee has developed an FDG-PET/CT digital reference object (DRO) that is a synthetic test object representing an FDG-PET image volume in the Digital Imaging and Communications in
Medicine (DICOM) format [9]. The DRO images in both PET and CT are based on the body phantom of National Electrical Manufacturers Association (NEMA) and International Electrotechnical Commission (IEC) [10]. Since the DRO is created synthetically with no random image noise, the DRO can be used as a reference standard to test SUV calculations. Pierce et al. used the DRO, which was smoothed by partial voxel computation in view of finite spatial resolution, to ensure the standardization of SUV computation in PET between medical image viewing workstations [11]. According to the Japanese guideline for oncological FDG-PET/CT imaging in 2009, a DRO-like digital phantom smoothed by a 3D Gaussian filter (3DGF) with a FWHM of $10 \mathrm{~mm}$ was used as a reference in order to define prerequisite image quality for detection of a 10$\mathrm{mm}$ hot sphere with SUV of 4 [12].

In the present study, we propose a use of the DRO smoothed by 3DGF with a FWHM of $17 \mathrm{~mm}$ $\left(\mathrm{DRO}_{17} \mathrm{~mm}\right)$ for the purpose of harmonizing SUVs in Tc-99m SPECT/CT imaging. Our phantom study using the aforementioned four state-of-the-art SPECT/CT systems to image NEMA phantom showed that a $10-\mathrm{mm}$ hot sphere was undetected and a 13- $\mathrm{mm}$ hot sphere was barely discernible, whereas all the scanners clearly depicted a $17-\mathrm{mm}$ sphere. Based on the detectable feature, we hypothesized that $\mathrm{DRO}_{17} \mathrm{~mm}$ could be used as a reference to determine the harmonization-specific imaging protocol as a digital phantom with a smooth of $10 \mathrm{~mm}$ which was used in the Japanese PET guideline [12]. The aim of this study was to demonstrate the feasibility of SUV harmonization among these SPECT/CT using $\mathrm{DRO}_{17 \mathrm{~mm}}$ as a reference standard.

\section{Methods}

Determination of Tc-99m concentration in NEMA phantom to simulate clinical Tc-99m SPECT/CT

In order to determine Tc-99m concentration enclosed in the NEMA body phantom, the following procedure was performed; first, SPECT/CT scans using an integrated SPECT/CT system (Discovery NM/CT 670pro, GE Healthcare) equipped with a low-energy high-resolution collimator were performed in 28 cancer patients $3 \mathrm{~h}$ after intravenous injection of $740 \mathrm{MBq}$ of Tc-99m hydroxymethylene diphosphonate (Tc-99m HMDP) at one of the institutions participating in the present study. The SPECT data obtained from routine clinical examinations were used in order to determine Tc-99m concentration in the NEMA phantom, which was approved by the Institutional Review Board (IRB) in the hospital. The IRB officially granted permission for this retrospective review of the imaging data and waived the need for obtaining informed consent from the patients. SPECT counts of the lower abdominal portion were measured in order to obtain the reference counting rates $(11.2 \pm$ 
3.5 kilo counts per second (kcps)) for the phantom study. Second, the body phantom in which six hot spheres $(10,13,17,22,28$, and $37 \mathrm{~mm})$ were embedded was filled with Tc-99m solution so that the spheres had a 4:1 radioactivity ratio compared with the background. At the beginning of the SPECT scan, the radioactivity concentration and the SPECT counting rate of the phantom were $36 \mathrm{kBq} / \mathrm{cc}$ and $22.8 \mathrm{kcps}$, respectively (Fig. 1). Then, 6-min SPECT/CT scans were performed repeatedly with an interval of $60 \mathrm{~min}$ for $12 \mathrm{~h}$. Based on the results of the correlation between the radioactivity concentration and the SPECT counting rate, the optimal radioactivity concentration for further phantom studies were determined.

\section{Calculation of calibration factor for SUV measurement using a cylindrical phantom}

A cylindrical phantom with a diameter of $160 \mathrm{~mm}$ and a height of $150 \mathrm{~mm}(3016 \mathrm{~mL})$ filled with Tc-99m solution of known activity concentration (approximately $25 \mathrm{MBq}$ ) was scanned for $6 \mathrm{~min}$. Data were reconstructed with 3D-OSEM with scatter and CT-based attenuation correction and were processed with various parameter settings including the pre-harmonized conditions used in each of the four SPECT/CT cameras. Basic performances of the SPECT/CT cameras were describe elsewhere [1], and the detailed imaging conditions and collimator configurations are shown in Table 1 . Parameter settings are comprised of the product of subset and iteration numbers (SI, range 40-160) and 3DGF (range 1.0-4.0 pixel (Infinia and Discovery; pixel size, $4.4 \mathrm{~mm}$ ) or $5-17 \mathrm{~mm}$ (Brightview and Symbia)). The processing of 3DGF in Philips Brightview XCT was performed using a commercially available software GI-PET

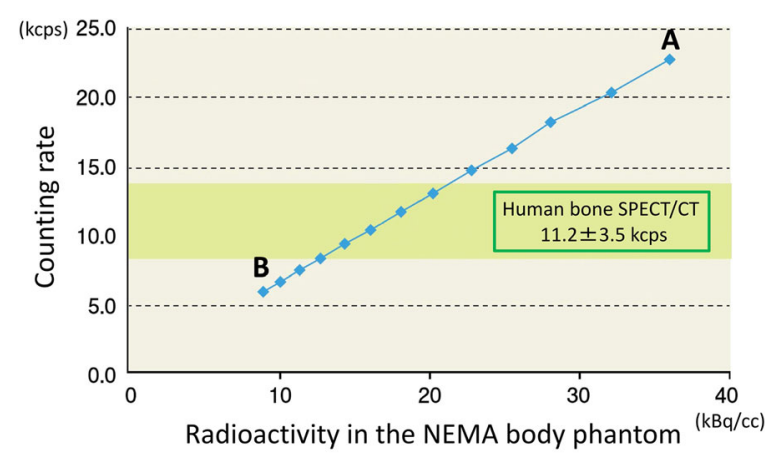

A: $36 \mathrm{kBq} / \mathrm{cc}, 22.8 \mathrm{kcps}$

B: $9 \mathrm{kBq} / \mathrm{cc}, 5.9 \mathrm{kcps}$

Fig. 1 Correlation of Tc-99m concentration of the NEMA phantom and counting rate in Discovery NM/CT 670 from the beginning $(A)$ to the end $(B)$ of the SPECT scan. There was a linear relationship between the radioactivity and counting rate. Tc-99m concentration for further phantom study was determined based on the counting rate of clinical bone SPECT/CT
(AZE Co., Ltd., Tokyo, Japan) because this filter option was not installed in any imaging workstation belonging to the institution with Philips Brightview XCT. Resolution recovery (RR) by compensating the distancedependent detector response was used.

SPECT/CT data in each reconstruction condition were analyzed using a commercially available software GIBONE (AZE Co., Ltd., Tokyo, Japan). With the software, slice thickness was automatically converted to be about $2 \mathrm{~mm}$ to allow isotropic voxel evaluation (Table 1). A circular ROI was drawn on the center of the cylindrical phantom in the central slice as well as in slices \pm 1 and $\pm 2 \mathrm{~cm}$ away, measuring SPECT count density (count/cc). The calibration factor was calculated as the ratio of actual radioactivity concentration (as measured by the dose calibrator) in the phantom at the time of scanning (ACC) to the measured SPECT count density per scan duration (MC), and we call this factor Becquerel calibration factor (BCF). Consequently, the BCF is calculated as:

$$
\mathrm{BCF}_{[\mathrm{Bq} / \mathrm{cps}]}=\frac{\mathrm{ACC}_{[\mathrm{Bq} / \mathrm{cc}]}}{\mathrm{MC}_{[\mathrm{count} / \mathrm{cc} \times 1 / \mathrm{sec}]}}
$$

The BCF should be dependent on the performance of SPECT/CT system and imaging conditions. The MC also should be affected by a scaling factor (multiplying pixel count in reconstruction with RR) in GE resolution modeling.

SUV conversion of NEMA body phantom image using BCF In order to simulate clinical Tc-99m SPECT/CT scans, the activity concentration levels in the background and spheres in the NEMA body phantom were set at 18 and $54 \mathrm{kBq} / \mathrm{cc}$, respectively (Fig. 1). The phantom was scanned for $6 \mathrm{~min}$ with the four different SPECT/CT systems. The phantom images were reconstructed in the same parameter setting as BCF images. The phantom data in each reconstruction condition were analyzed using the same software as BCF data. Six different target ROIs, whose diameters were equal to the physical inner diameters of the hot spheres, were placed on the target slice. The SUV is calculated as:

$$
\mathrm{SUV}=\mathrm{BCF}_{[\mathrm{Bq} / \mathrm{cps}]} \times \mathrm{MC}_{[\text {count/cc g } 1 / \text { sec }]} \times \frac{\text { Body weight }_{[\mathrm{g}]}}{\text { Injected activity }_{[\mathrm{Bq}]}}
$$

In this phantom study, the reciprocal of body weight per injected dose was $9000 \mathrm{~Bq} / \mathrm{g}$ so that background SUV was 1. Regarding calculation of SUVs, 10-, 13-, 17-, 22-, 28-, and 37-mm circular ROIs were drawn exactly on the corresponding spheres in the central slice by following the CT boundaries of the fused SPECT/CT images. Then, $\mathrm{SUV}_{\text {peak }}, \mathrm{SUV}_{\mathrm{max}}$, and the mean SUV $\left(\mathrm{SUV}_{\text {mean }}\right)$ for the spheres were measured. Peak SUV 
Table 1 Imaging conditions and collimator configurations regarding the four state-of-the-art SPECT/CT systems

\begin{tabular}{|c|c|c|c|c|}
\hline & Brightview XCT & Discovery NM/CT 670 & Infinia Hawkeye 4 & Symbia T6 \\
\hline \multicolumn{5}{|l|}{ Imaging condition } \\
\hline \multicolumn{5}{|l|}{ Step and shoot image acquisition } \\
\hline No. of step & 30 & 30 & 30 & 30 \\
\hline Rotation angle & 6 & 6 & 6 & 6 \\
\hline No. of projection & 60 & 60 & 60 & 60 \\
\hline Scan orbit & body contour & body contour & body contour & body contour \\
\hline \multicolumn{5}{|l|}{ Size for image acquisition } \\
\hline Matrix $(x, y, z)$ & $132,132,132$ & $128,128,128$ & $128,128,128$ & $128,128,128$ \\
\hline Pixel size and slice thickness (mm) & 4.7 & 4.4 & 4.4 & 4.8 \\
\hline Smoothing filter & 3D Gaussian & 3D Gaussian & 3D Gaussian & 3D Gaussian \\
\hline \multicolumn{5}{|l|}{ Reconstructed image for $\mathrm{ROI}$ analysis } \\
\hline Matrix $(x, y, z)$ & $256,256,203$ & $256,256,207$ & $256,256,130$ & $256,256,187$ \\
\hline Pixel size and slice thickness (mm) & 2.0 & 2.0 & 2.2 & 2.0 \\
\hline \multicolumn{5}{|l|}{ Energy window } \\
\hline Main & $140.5 \mathrm{keV} \pm 10 \%$ & $140.5 \mathrm{keV} \pm 10 \%$ & $140 \mathrm{keV} \pm 10 \%$ & $140 \mathrm{keV} \pm 10 \%$ \\
\hline Sub & N.A. & $120 \mathrm{keV} \pm 5 \%$ & $120 \mathrm{keV} \pm 5 \%$ & $120 \mathrm{keV} \pm 5 \%$ \\
\hline Attenuation correction & CT-based & CT-based & CT-based & CT-based \\
\hline Scatter correction & ESSE & DEW & DEW & DEW \\
\hline \multicolumn{5}{|l|}{ Collimator } \\
\hline Type & CHR & LEHR & LEHR & LEHR \\
\hline No. of holes (thousand) & 40.2 & 86.3 & 86.3 & 148 \\
\hline Hole shape & Hexagon & Hexagon & Hexagon & Hexagon \\
\hline Hole length (mm) & 48 & 35 & 35 & 24.1 \\
\hline Septal thickness (mm) & 0.15 & 0.20 & 0.20 & 0.16 \\
\hline Hole diameter across the flats (mm) & 2.03 & 1.50 & 1.50 & 1.11 \\
\hline
\end{tabular}

ESSE effective source scatter estimation method. DEW dual-energy window method, CHR cardiac high-resolution collimator, LEHR low-energy high-resolution collimator, N.A. not applicable

represents the average SUV obtained within a 1-cc sphere of region of interest (ROI) centered on a highest voxel of the target area.

\section{Harmonization of SUVs using $\mathrm{DRO}_{17 \mathrm{~mm}}$}

Simulated images of original DRO and $\mathrm{DRO}_{17} \mathrm{~mm}$ are shown in Fig. 2. SUV mean $\mathrm{SUV}_{\text {peak }}$, and $\mathrm{SUV}_{\text {max }}$ of the six spheres in $\mathrm{DRO}_{17} \mathrm{~mm}$ are described in Table 2. As a measure of harmonization of reconstruction conditions, the root mean square error (RMSE) was measured; RMSE is the square root of the variance in $S_{U V}$ mean of the six spheres between $\mathrm{DRO}_{17} \mathrm{~mm}$ and actual phantom images obtained with the SPECT/CT cameras. Therefore, RMSE is measured as:
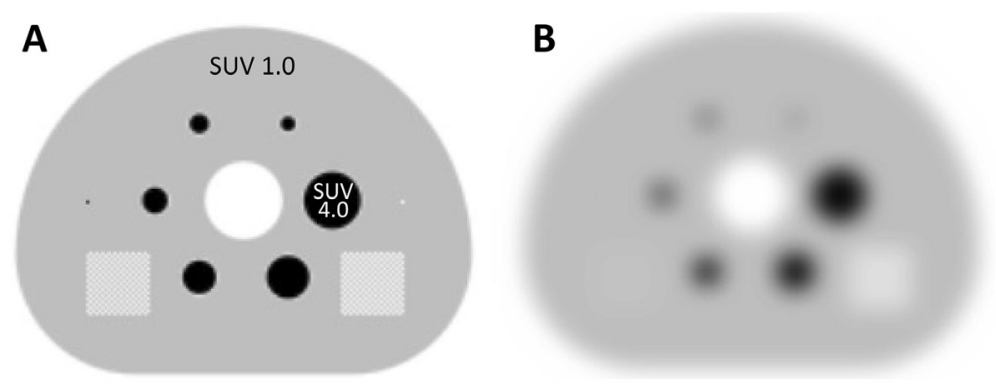

Fig. 2 a Phantom configuration of a digital reference object (DRO) and $\mathbf{b}$ DRO filtered by a 17-mm Gaussian filter (DRO 17 mm). The square boxes in DRO are by design (not used in the present study) 


$$
\mathrm{RMSE}=\sqrt{\frac{1}{6} \sum_{i=10,13,17,22,88,37 \mathrm{~mm}}\left(\mathrm{SUV}_{\text {mean in phantom, }, i}-\mathrm{SUV}_{\text {mean in DRO17mm, i }}\right)^{2}}
$$

Reconstruction conditions according to the manufacturers' recommendation or the policies of their own departments are shown in Table 3. SUV $\mathrm{Sean}_{\text {men }}$ of the hot spheres and RMSE in the pre-harmonized conditions are also shown in Table 3.

RMSE was calculated in the following conditions: $S I$, range 40-140; 3DGF, range 1.0-4.0 pixel (Infinia and Discovery) or 5-17 mm (Brightview and Symbia). Then, for every scanner examined, settings were found that showed a clear optimum for harmonization. In both preand post-harmonized conditions, coefficient of variation $(\mathrm{COV})$ of $\mathrm{SUV}_{\text {mean }}, \mathrm{SUV}_{\text {max }}$, and $\mathrm{SUV}_{\text {peak }}$ between the four scanners were calculated.

\section{Results}

\section{Tc-99m concentration for phantom study}

As shown in Fig. 1, Tc-99m concentration in the NEMA body phantom had linear correlation with the acquisition counting rate. The counting rates in human bone SPECT/CT $(11.2 \pm 3.5 \mathrm{kcps})$ were equivalent to Tc- $99 \mathrm{~m}$ concentration of $12.8-22.9 \mathrm{kBq} / \mathrm{ml}$. Therefore, the activity concentration levels in the background and spheres for further evaluation were set at 18 and $54 \mathrm{kBq} / \mathrm{cc}$, resulting in mean activity concentration of the entire phantom of approximately $18.3 \mathrm{kBq} / \mathrm{cc}$.

\section{BCF measurement}

Table 4 shows the distribution of BCF among SPECT/ CT systems with different reconstruction conditions. The difference in BCF value was small between BrightView and Symbia and between Infinia and Discovery. A scaling factor seemed to affect the BCF. Reconstruction

Table 2 SUV values derived from a digital reference object smoothed by a 17-mm Gaussian filter ( $\mathrm{DRO}_{17} \mathrm{~mm}$ )

\begin{tabular}{llcl}
\hline \multicolumn{4}{l}{ Quantitative metrics } \\
\hline & \multicolumn{2}{l}{ Quantitative metrics } \\
& SUV $_{\text {mean }}$ & SUV & \\
Spheak & & SUV \\
& & & \\
10 & 1.18 & 1.17 & 1.20 \\
13 & 1.36 & 1.38 & 1.42 \\
17 & 1.67 & 1.77 & 1.86 \\
22 & 2.12 & 2.40 & 2.53 \\
28 & 2.55 & 3.14 & 3.29 \\
37 & 2.94 & 3.82 & 3.91 \\
\hline
\end{tabular}

conditions did not significantly affect the BCF (approximately less than $3 \%$ of mean value).

\section{Effects of reconstruction parameter settings on SUVs and RMSE}

Figure 3 shows RMSE for the four SPECT/CT systems. RMSE was lowest in the following reconstruction conditions: SI of 100 and 3DGF of $13 \mathrm{~mm}$ for Brightview XCT (RMSE $=0.115$ ); SI of 160 and 3DGF of 3 pixels for Discovery NM/CT (RMSE $=0.085$ ); SI of 60 and 3DGF of 2 pixels for Infinia (RMSE $=0.102$ ); and SI of 140 and 3DGF of $15 \mathrm{~mm}$ for Symbia (RMSE $=0.117$ ). It should be noted that the minimum RMSE was below 0.12 for each harmonized setting.

\section{$\mathrm{SUV}_{\text {mean }}, \mathrm{SUV}_{\text {max }}$ and SUV $\mathrm{V}_{\text {peak }}$ in both pre- and post-harmonized conditions}

Figure 4 shows $\mathrm{SUV}_{\text {mean }}$, $\mathrm{SUV}_{\text {max }}$, and $\mathrm{SUV}_{\text {peak }}$ of the hot spheres in both pre- and post-harmonized conditions. Table 5 shows COVs of these metrics between the four SPECT/CT systems. In pre-harmonized conditions, COVs were greater than $10 \%$ for all metrics in the 17 -, 22-, and 28-mm spheres, $\mathrm{SUV}_{\max }$ in the 13-mm sphere and $\mathrm{SUV}_{\text {mean }}$ in the $37-\mathrm{mm}$ sphere. In contrast, all

Table 3 Reconstruction conditions according to the manufacturers' recommendation or the policies of their own departments

\begin{tabular}{lllll}
\hline \multicolumn{5}{c}{ SPECT/CT scanner } \\
\hline & BrightView & Discovery & Infinia & Symbia \\
Reconstruction parameter & & & & \\
Subset & 8 & 10 & 10 & 10 \\
Iteration & 10 & 10 & 10 & 10 \\
Filter & Gaussian & Gaussian & Gaussian & Gaussian \\
Cutoff value & $15 \mathrm{~mm}$ & 2.5 pixel & 2.5 pixel & $9 \mathrm{~mm}$ \\
Resolution recovery & Astonish & Evolution & Evolution & FLASH 3D \\
SUV ${ }_{\text {mean }}$ of the spheres & & & & \\
$10 \mathrm{~mm}$ & 1.08 & 1.25 & 0.98 & 1.06 \\
$13 \mathrm{~mm}$ & 1.14 & 1.48 & 1.28 & 1.37 \\
$17 \mathrm{~mm}$ & 1.38 & 1.73 & 1.73 & 1.99 \\
$22 \mathrm{~mm}$ & 1.81 & 2.19 & 1.92 & 2.52 \\
$28 \mathrm{~mm}$ & 2.41 & 2.96 & 2.42 & 3.08 \\
$37 \mathrm{~mm}$ & 2.86 & 3.21 & 2.71 & 3.50 \\
RMSE & 0.20 & 0.20 & 0.13 & 0.40 \\
\hline
\end{tabular}


Table 4 Distribution of BCF among SPECT/CT systems with different reconstruction conditions

\begin{tabular}{lllll}
\hline \multicolumn{5}{l}{ SPECT/CT scanner } \\
\hline & BrightView & Discovery $^{\text {a }}$ & Infinia $^{\text {a }}$ & Symbia \\
Mean & 5309 & 1617 & 1538 & 4914 \\
Standard deviation & 35 & 5 & 48 & 32 \\
$\begin{array}{l}\text { Relative standard } \\
\text { deviation (\%) }\end{array}$ & 0.7 & 0.3 & 3.1 & 0.7 \\
\hline
\end{tabular}

${ }^{\mathrm{a} A}$ scaling factor is involved with the values

metrics except $\mathrm{SUV}_{\max }$ in the 17-mm sphere yielded less than $10 \%$ of COVs after harmonization.

\section{Discussion}

Recent advances in SPECT/CT technologies allowed major manufacturers to mass-produce commercial SPECT/CT systems for clinical application of not only SPECT/CT fusion imaging but also fully quantitative SPECT imaging. Although PET/CT has become an important diagnostic tool quantifying tracer uptake, only a small number of PET tracers have yet been approved in clinical practice. In contrast, there have already been various kinds of available radiopharmaceuticals labeled with single-photon emitters; much focus is being placed on the value of quantitative SPECT/CT $[4,5,13-16]$.
Especially, clinical application of quantitative SPECT/CT using bone-seeking radiotracers is highly expected as shown in a successful report on the use of F-18 fluorine PET for prognostic assessment [17] as well as the accumulated evidences of quantitative planar bone scintigraphy in prostate cancer [18]. The bone scan index (BSI) $[19,20]$, which quantifies the total bone metastatic burden relative to the total skeletal mass on two-dimensional images, is getting wider acceptance as a biomarker for predicting survival in patients with prostate cancer [21-26]. However, there are substantial false-positive and false-negative findings when evaluating bone metastasis without SPECT/CT [27-31]. In addition, quantifying tracer accumulation on a per-lesion basis is limited by the projection of several overlying structures in a planar image. For instance, uptake in the sternum may contain some amounts of uptake in the thoracic spine in an anterior view of planar image, and quantitative analysis would therefore be difficult especially when metastasis occurs in these bones. We envisaged that harmonizing SUVs using the DRO could be applied to multicenter clinical trials using Tc-99m SPECT/CT; in particular, harmonized SUVs in bone SPECT/CT may become an alternative choice to BSI. In addition, the harmonization method might be utilized to

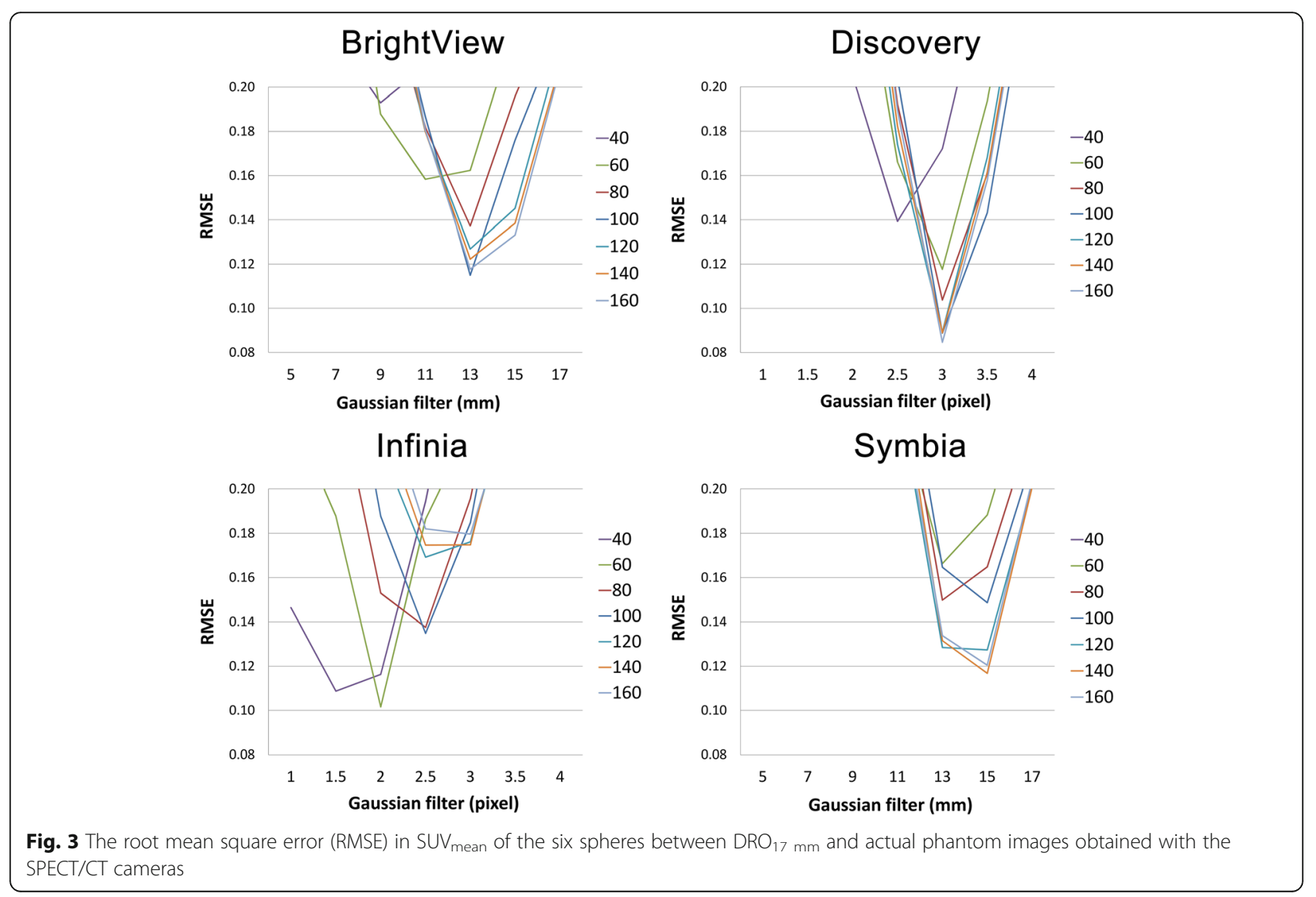




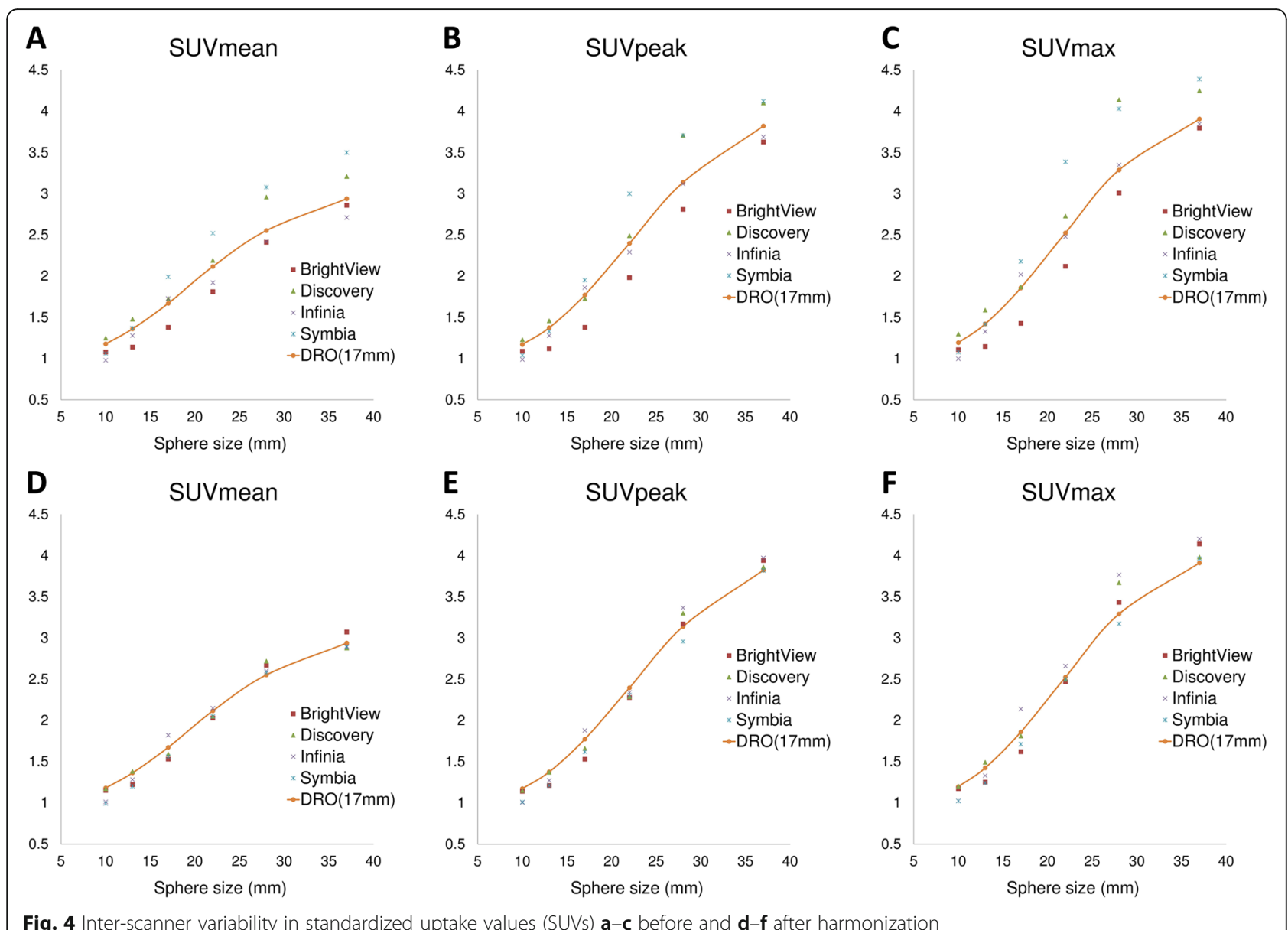

reduce inter-scanner variability in measurement of SPECT/CT-derived absorbed doses in a variety of "theranostics" situations such as Tc-99m MAA SPECT/CT in Y-90 microsphere therapy, I-131 SPECT/CT in thyroid cancer therapy, and Lu-177-PSMA in prostate cancer therapy [32-34].

We used DRO for harmonization instead of a two-step approach of assessing how close each scanner can get to true SUV (i.e., SUV =4) and then harmonizing to the

Table 5 Coefficient of variations (COVs) of SUVs between the four SPECT/CT systems

\begin{tabular}{lllllll}
\hline & \multicolumn{3}{c}{ Pre-harmonization } & \multicolumn{3}{l}{ Post-harmonization } \\
\hline Spheres & SUV $_{\text {mean }}$ & SUV $_{\text {peak }}$ & SUV $_{\text {max }}$ & SUV $_{\text {mean }}$ & SUV $_{\text {peak }}$ & SUV $_{\text {max }}$ \\
$10 \mathrm{~mm}$ & 9.00 & 8.23 & 9.81 & 7.47 & 6.42 & 7.48 \\
$13 \mathrm{~mm}$ & 9.46 & 9.38 & 11.57 & 5.51 & 5.17 & 7.54 \\
$17 \mathrm{~mm}$ & 12.70 & 12.52 & 14.90 & 7.05 & 7.66 & 10.77 \\
$22 \mathrm{~mm}$ & 12.99 & 15.20 & 17.30 & 2.24 & 1.00 & 2.94 \\
$28 \mathrm{~mm}$ & 11.24 & 11.63 & 12.93 & 2.11 & 4.85 & 6.56 \\
$37 \mathrm{~mm}$ & 10.02 & 5.82 & 6.21 & 2.67 & 1.55 & 2.51 \\
\hline
\end{tabular}

lower common denominator based on the following reasons:

1. We found that both $\mathrm{SUV}_{\text {max }}$ and $\mathrm{SUV}_{\text {peak }}$ fluctuated when acquisition time or phantom radioactivity was changed, probably due to image noise. In contrast, $\mathrm{SUV}_{\text {mean }}$ did not (data not shown). Therefore, we thought that $\mathrm{SUV}_{\max }$ and $\mathrm{SUV}_{\text {peak }}$ are not suitable parameters for harmonization in terms of test-retest reproducibility.

2. Although $\mathrm{SUV}_{\text {mean }}$ may be used for harmonization because of being unsusceptible to image noise, it never reached the uptake value of 4 even for the largest sphere $(37 \mathrm{~mm})$ due to partial volume effect. Hence, it seems impractical to assess how close each scanner can reach $S_{U V}$ mean of 4. Instead, DRO was smoothed to match SUVs of the targets in each of SPECT/CT systems with the corresponding SUVs in DRO.

3. Presetting DRO as a reference allows a variety of institutions to easily join the harmonization projects without any revisions of previously harmonized protocols in enrolled institutions, whereas the 
two-step approach seems complex when many scanners need to be harmonized.

It is important to know how accurate the current SPECT/CT technologies can be in terms of quantitation. It goes without saying, however, that even the state-ofthe-art SPECT/CT systems are less reliable than general $\mathrm{PET} / \mathrm{CT}$ systems especially in quantifying small lesions due to limited detector sensitivity and intrinsic spatial resolution; not surprisingly, a $10-\mathrm{mm}$ hot sphere was undetected with any of the four SPECT/CT systems under all reconstruction conditions, and a 13- $\mathrm{mm}$ hot sphere was barely discernible in most of the reconstructed SPECT images (data not shown). Based on the fact that point spread function or line spread function of the SPECT detectors, which represents image blurring due to finite spatial resolution, can be geometrically approximated by Gaussian function; $\mathrm{DRO}_{10} \mathrm{~mm}$ or $\mathrm{DRO}_{13 \mathrm{~mm}}$ was considered unsuitable to serve as a reference image. In contrast, the actual hot spheres measuring at least $17 \mathrm{~mm}$ were clearly observed irrespective of reconstruction conditions and SPECT/CT systems. Therefore, $\mathrm{DRO}_{17} \mathrm{~mm}$ was chosen as a reference image in the present study.

3DGF was used throughout the harmonization instead of using another filter such as Butterworth and Hanning filters. This is not only because $\mathrm{DRO}_{17} \mathrm{~mm}$ was generated with Gaussian filter, but because measurement of BCF with Gaussian filter was more stable than that with Butterworth or Hanning filter when changing reconstruction parameters such as $S I$ and cutoff value of these filters (data not shown).

We found that the lowest RMSE value was obtained with 3DGF of 8.8 to $15 \mathrm{~mm}$ and SI of 60 or 160 (Fig. 3). The results were partially different from the European Association of Nuclear Medicine (EANM) practice guidelines and recommendations of the camera manufacturers [35], which indicates a need for harmonization-specific imaging protocol. As shown in Fig. 4 and Table 5, interscanner variability in SUVs among the state-of-the-art SPECT/CT systems was clearly decreased after the proposed harmonization procedure. In this context, we propose the use of a digital phantom developed by QIBA for harmonizing SUVs in multicenter trials.

In PET/CT, the EANM guidelines do not positively recommend the use of resolution recovery for quantitative assessment in multicenter studies due to Gibbs artifact [36]. We observed small amount of uptake biased to a peripheral side of the sphere in the larger spheres (e.g., 37-mm sphere), probably due to Gibbs phenomenon. This effect possibly resulted in a slight elevation of $\mathrm{SUV}_{\max }$ over true value (i.e., $\mathrm{SUV}=4$ ) as shown in Fig. 4. Although resolution recovery was responsible for the overshoot, the lack of resolution recovery significantly underestimated SUVs. For instance, $S U V_{\text {max }}$ and $S U V_{\text {mean }}$ of a $37-\mathrm{mm}$ sphere without resolution recovery were about 3.3 and 2.5 , respectively. In light of the principle of photon detection with collimator-dependent SPECT systems, it is reasonable to compensate the distance-dependent detector response for lesion-based quantitative assessment. At present, we consider that resolution recovery should be used at the sacrifice of the small amount of the overshoot.

In the present study, the radioactivity of the NEMA phantom was determined on the basis of the bone SPECT data under a 6-min acquisition protocol. According to the Japanese technological guidelines on nuclear imaging, bone SPECT data should be collected for 5-6 $\mathrm{min} /$ bed [37]. On the other hand, the EANM practice guidelines indicate the acquisition time of 10 $30 \mathrm{~min} / \mathrm{bed}$ [35]. The difference may be due to the fact that radioactive dose administered to patients undergoing bone scintigraphy is different between Japan and Europe (mean dose, 740 vs $500 \mathrm{MBq}$ ). In addition, considering the difference in body weight and height between Japanese (light and short) and European people (heavy and tall), 6 min SPECT acquisition for Japanese patients would be equivalent to $10 \mathrm{~min}$ or more acquisition for European patients in terms of SPECT counts per bed position.

There are several limitations in this study. First, $\mathrm{DRO}_{17} \mathrm{~mm}$ has no absolute and universal significance as a reference image. It does not seem to be necessary to smooth to a level which matches the sphere size. In other words, $\mathrm{DRO}_{14 \mathrm{~mm}}, \mathrm{DRO}_{15 \mathrm{~mm}}$, or $\mathrm{DRO}_{16} \mathrm{~mm}$ might serve as better references. In our preliminary study, $\mathrm{DRO}_{17} \mathrm{~mm}$ was arbitrarily determined as a reference. However, it is worth noting that the minimum RMSE was below 0.12 for each harmonized setting (Fig. 3) and that the harmonized SUV curves as a function of sphere size are close to the curve of $\mathrm{DRO}_{17} \mathrm{~mm}$ as shown in Fig. 4. Hence, we considered that $\mathrm{DRO}_{17} \mathrm{~mm}$ could be a suboptimal reference for a multicenter study and that $\mathrm{DRO}_{14 \mathrm{~mm}}, \mathrm{DRO}_{15 \mathrm{~mm}}$, and $\mathrm{DRO}_{16 \mathrm{~mm}}$ may also be references for another multicenter study. In any case, it seems important to specify which DRO is regarded as a reference together with RMSE for each scanner. Our results suggest that RMSE of 0.12 may serve as an index of appropriateness of harmonization. Second, background and cold regions were not focused on in this study. This study was intended for a variety of multicenter SPECT studies such as bone SPECT/CT in which quantitation of background or cold regions would be unnecessary. In other words, the results of our study should not be applied to myocardial or cerebral perfusion SPECT/CT in which decrease in tracer uptake has significant impact on treatment strategy. In this context, we believe that our study is the first step to expand the 
use of DRO by QIBA for the future of quantitation using SPECT/CT. Finally, the currently available DRO has a contrast of 4:1. Therefore, we collected SPECT data of the NEMA phantom with 4:1 concentration ratio. Whether the results would be applicable for other contrast remains unknown. Examining this issue is one of the top priorities for further research.

\section{Conclusions}

In the present study, the DRO smoothed by 3DGF with a FWHM of $17 \mathrm{~mm}$ was used for the purpose of harmonizing SUVs in Tc-99m SPECT/CT imaging. SUVs generated according to the manufacturers' recommendation or the policies of their own departments had substantial inter-scanner variability, indicating a need for harmonization-specific imaging protocols. Our harmonization clearly reduced interscanner variability in all metrics except $\mathrm{SUV}_{\max }$ in the $17-\mathrm{mm}$ sphere with less than $10 \%$ of COVs. A digital phantom developed by QIBA would be useful for harmonizing SUVs in multicenter trials.

\begin{abstract}
Abbreviations
3DGF: Three-dimensional Gaussian filter; BCF: Becquerel calibration factor; BSI: Bone scan index; CHR: Cardiac high-resolution collimator;

COV: Coefficient of variation; DEW: Dual-energy window method; DICOM: Digital imaging and communications in medicine; DRO: Digital reference object; ESSE: Effective source scatter estimation method; FDG: Fluorodeoxyglucose; HMDP: Hydroxymethylene diphosphonate; IEC: International Electrotechnical Commission; LEHR: Low-energy highresolution collimator; NEMA: National Electrical Manufacturers Association; PET/CT: Positron emission tomography/computed tomography; QIBA: Quantitative imaging biomarker alliance; RMSE: Root mean square error; ROI: Region of interest; SPECT/CT: Single-photon emission computed tomography/computed tomography; SUV: Standardized uptake value; VOl: Volume of interest
\end{abstract}

\section{Acknowledgements}

We would like to thank nuclear medicine staff at the five hospitals participating in this study.

\section{Funding}

None.

\section{Authors' contributions}

TN contributed to the study design, analyzing of the data, drafting of the manuscript, and critical revision of the manuscript. HD contributed to the study design, preparation of the study, analyzing of the data. YY, $\mathrm{TI}, \mathrm{KM}, \mathrm{TO}$ YO, NY, KS, and RT contributed to phantom data collection. MJ contributed to the study design and critical revision of the manuscript. All authors read and approved the final manuscript.

\section{Competing interests}

The authors declare that they have no competing interests.

\section{Ethics approval}

The SPECT data obtained from routine clinical examinations were used in order to determine Tc-99m concentration in the NEMA phantom, which was approved by the Institutional Review Board (IRB) in the hospital. The IRB officially granted permission for this retrospective review of the imaging data and waived the need for obtaining informed consent from the patients.

\section{Publisher's Note}

Springer Nature remains neutral with regard to jurisdictional claims in published maps and institutional affiliations.

\section{Author details}

'Department of Radiology, Keio University School of Medicine, 35 Shinanomachi, Shinjuku-ku, Tokyo 160-8582, Japan. ${ }^{2}$ Department of Radiological Technology, Gunma Prefectural College of Health Sciences, 323-1 Kamioki-machi, Maebashi, Gunma 371-0052, Japan. ${ }^{3}$ Department of Radiology, Shimane University Hospital, 89-1 Enya-cho, Izumo, Shimane 693-8501, Japan. ${ }^{4}$ Department of Radiology, Chiba University Hospital, 1-8-1 Inohana, Chuo-ku, Chiba-shi, Chiba 260-8677, Japan. ${ }^{5}$ Department of Radiology, Kochi Medical Hospital, 2125-1 Ike, Kochi-shi, Kochi 781-8555, Japan. ${ }^{6}$ Department of Radiology, Tsugaru General Hospital, 12-3 Iwaki-machi, Goshogawara-shi, Aomori 037-0074, Japan. ${ }^{7}$ Department of Radiological Sciences, Tokyo Metropolitan University, 7-2-10 Higashiogu, Arakawa-ku, Tokyo 116-8551, Japan.

Received: 31 March 2017 Accepted: 6 June 2017

Published online: 20 June 2017

\section{References}

1. Seret A, Nguyen D, Bernard C. Quantitative capabilities of four state-of-the-art SPECT-CT cameras. EJNMMI Res. 2012;2:45.

2. Hughes T, Celler A. A multivendor phantom study comparing the image quality produced from three state-of-the-art SPECT-CT systems. Nucl Med Commun. 2012;33:663-70.

3. Zeintl J, Vija AH, Yahil A, Hornegger J, Kuwert T. Quantitative accuracy of clinical ${ }^{99 \mathrm{~m}} \mathrm{TC}$ SPECT/CT using ordered-subset expectation maximization with 3-dimensional resolution recovery, attenuation, and scatter correction. J Nucl Med. 2010;51:921-8.

4. Suh MS, Lee WW, Kim YK, Yun PY, Kim SE. Maximum standardized uptake value of $(99 \mathrm{~m}) \mathrm{Tc}$ hydroxymethylene diphosphonate SPECT/CT for the evaluation of temporomandibular joint disorder. Radiology. 2016;280:890-6.

5. Tofuku K, Koga H, Komiya S. The diagnostic value of single-photon emission computed tomography/computed tomography for severe sacroiliac joint dysfunction. Eur Spine J. 2015:24:859-63.

6. Beck M, Sanders JC, Ritt P, Reinfelder J, Kuwert T. Longitudinal analysis of bone metabolism using SPECT/CT and (99m)Tc-diphosphonopropanedicarboxylic acid: comparison of visual and quantitative analysis. EJNMMI Res. 2016:6:60.

7. Lasnon C, Desmonts C, Quak E, Gervais R, Do P, Dubos-Arvis C, et al. Harmonizing SUVS in multicentre trials when using different generation PET systems: prospective validation in non-small cell lung cancer patients. Eur J Nucl Med Mol Imaging. 2013;40:985-96

8. Makris NE, Huisman MC, Kinahan PE, Lammertsma AA, Boellaard R. Evaluation of strategies towards harmonization of FDG PET/CT studies in multicentre trials: comparison of scanner validation phantoms and data analysis procedures. Eur J Nucl Med Mol Imaging. 2013;40:1507-15.

9. Quantitative imaging biomarker alliance. QIBA Profile. FDG-PET/CT for Response to Cancer Therapy 2016-11-18. http://qibawikirsnaorg/images/1/ 1f/QIBA_FDG-PET_Profile_v113pdf. Accessed 24 Feb 2017.

10. Daube-Witherspoon ME, Karp JS, Casey ME, Difilippo FP, Hines H, Muehllehner G, et al. PET performance measurements using the NEMA NU 2-2001 standard. J Nucl Med. 2002:43:1398-409.

11. Pierce 2nd LA, Elston BF, Clunie DA, Nelson D, Kinahan PE. A digital reference object to analyze calculation accuracy of PET standardized uptake value. Radiology. 2015;277:538-45.

12. Fukukita H, Senda M, Terauchi T, Suzuki K, Daisaki H, Matsumoto K, et al. Japanese guideline for the oncology FDG-PET/CT data acquisition protocol: synopsis of Version 1.0. Ann Nucl Med. 2010;24:325-34.

13. He W, Zhai W, Steien S, Vagstad T, Skjarmoen B, Olsen E, et al. A novel quantification of ${ }^{99 \mathrm{~m}} \mathrm{Tc}-\mathrm{MDP}$ concentration and ${ }^{18} \mathrm{~F}$-FDG PET/CT metabolism in lumbar disc herniation patients. J Nucl Med. 2016;57(Suppl 2):1817.

14. Golfam M, Ruddy T, Wells RG. Feasibility of SPECT SUV to quantify 123I-MIBG cardiac uptake in heart failure patients. J Nucl Med. 2016;57(Suppl 2):228.

15. Rowe SP, Vicente E, Anizan N, Wang H, Leal JP, Lodge MA, et al. Repeatability of radiotracer uptake in normal abdominal organs with ${ }^{111}$ In-pentetreotide quantitative SPECT/CT. J Nucl Med. 2015;56:985-8.

16. Hanaoka K, Hosono M, Tatsumi Y, Ishii K, Im S-W, Tsuchiya N, et al. Heterogeneity of intratumoral ${ }^{111}$ In-ibritumomab tiuxetan and ${ }^{18} \mathrm{~F}-\mathrm{FDG}$ 
distribution in association with therapeutic response in radioimmunotherapy for B-cell non-Hodgkin's lymphoma. EJNMMI Res. 2015;5:1.

17. Etchebehere EC, Araujo JC, Fox PS, Swanston NM, Macapinlac HA, Rohren EM. Prognostic factors in patients treated with 223Ra: the role of skeletal tumor burden on baseline 18F-fluoride PET/CT in predicting overall survival. J Nucl Med. 2015;56:1177-84.

18. Zafeirakis A. Scoring systems of quantitative bone scanning in prostate cancer: historical overview, current status and future perspectives. Hell J Nucl Med. 2014;17:136-44.

19. Ulmert D, Kaboteh R, Fox JJ, Savage C, Evans MJ, Lilja H, et al. A novel automated platform for quantifying the extent of skeletal tumour involvement in prostate cancer patients using the bone scan index. Eur Urol. 2012;62:78-84.

20. Imbriaco M, Larson SM, Yeung HW, Mawlawi OR, Erdi Y, Venkatraman ES, et al. A new parameter for measuring metastatic bone involvement by prostate cancer: the bone scan index. Clin Cancer Res. 1998:4:1765-72.

21. Miyoshi Y, Yoneyama S, Kawahara T, Hattori Y, Teranishi J, Kondo K, et al. Prognostic value of the bone scan index using a computer-aided diagnosis system for bone scans in hormone-naive prostate cancer patients with bone metastases. BMC Cancer. 2016;16:128.

22. Reza M, Bjartell A, Ohlsson M, Kaboteh $R$, Wollmer P, Edenbrandt $L$, et al. Bone scan index as a prognostic imaging biomarker during androgen deprivation therapy. EJNMMI Res. 2014;4:58.

23. Armstrong AJ, Kaboteh R, Carducci MA, Damber JE, Stadler WM, Hansen M, et al. Assessment of the bone scan index in a randomized placebocontrolled trial of tasquinimod in men with metastatic castration-resistant prostate cancer (mCRPC). Urol Oncol. 2014;32:1308-16.

24. Mitsui $Y$, Shiina $H$, Yamamoto $Y$, Haramoto $M$, Arichi $N$, Yasumoto $H$, et al. Prediction of survival benefit using an automated bone scan index in patients with castration-resistant prostate cancer. BJU Int. 2012;110:E628-34.

25. Dennis ER, Jia X, Mezheritskiy IS, Stephenson RD, Schoder H, Fox JJ, et al. Bone scan index: a quantitative treatment response biomarker for castration-resistant metastatic prostate cancer. J Clin Oncol. 2012;30:519-24

26. Sabbatini $\mathrm{P}$, Larson $\mathrm{SM}$, Kremer $\mathrm{A}$, Zhang ZF, Sun $\mathrm{M}$, Yeung $\mathrm{H}$, et al. Prognostic significance of extent of disease in bone in patients with androgen-independent prostate cancer. J Clin Oncol. 1999;17:948-57.

27. Palmedo H, Marx C, Ebert A, Kreft B, Ko Y, Turler A, et al. Whole-body SPECT/CT for bone scintigraphy: diagnostic value and effect on patient management in oncological patients. Eur J Nucl Med Mol Imaging. 2014:41:59-67.

28. Helyar V, Mohan HK, Barwick T, Livieratos L, Gnanasegaran G, Clarke SE, et al. The added value of multislice SPECT/CT in patients with equivocal bony metastasis from carcinoma of the prostate. Eur J Nucl Med Mol Imaging 2010;37:706-13.

29. Utsunomiya D, Shiraishi S, Imuta M, Tomiguchi S, Kawanaka K, Morishita S, et al. Added value of SPECT/CT fusion in assessing suspected bone metastasis: comparison with scintigraphy alone and nonfused scintigraphy and CT. Radiology. 2006;238:264-71.

30. Romer W, Nomayr A, Uder M, Bautz W, Kuwert T. SPECT-guided CT for evaluating foci of increased bone metabolism classified as indeterminate on SPECT in cancer patients. J Nucl Med. 2006:47:1102-6.

31. Ogata Y, Nakahara T, Ode K, Matsusaka Y, Katagiri M, Iwabuchi Y, et al. 3D SPECT/CT fusion using image data projection of bone SPECT onto 3D volume-rendered CT images: feasibility and clinical impact in the diagnosis of bone metastasis. Ann Nucl Med. 2017;31:304-14.

32. Garin $E$, Rolland $Y$, Laffont $S$, Edeline J. Clinical impact of ${ }^{99 \mathrm{~m} T c-M A A ~ S P E C T /}$ CT-based dosimetry in the radioembolization of liver malignancies with ${ }^{90} \mathrm{Y}$ loaded microspheres. Eur J Nucl Med Mol Imaging. 2016;43:559-75.

33. Kabasakal L, AbuQbeitah M, Aygun A, Yeyin N, Ocak M, Demirci E, et al. Pre-therapeutic dosimetry of normal organs and tissues of (177)Lu-PSMA-617 prostate-specific membrane antigen (PSMA) inhibitor in patients with castration-resistant prostate cancer. Eur J Nucl Med Mol Imaging. 2015:42:1976-83

34. Chen M-K, Cheng DW. What is the role of dosimetry in patients with advanced thyroid cancer? Curr Opin Oncol. 2015:27:33-7.

35. Van den Wyngaert T, Strobel K, Kampen WU, Kuwert T, van der Bruggen W, Mohan HK, et al. The EANM practice guidelines for bone scintigraphy. Eur J Nucl Med Mol Imaging. 2016;43:1723-38.
36. Boellaard R, Delgado-Bolton R, Oyen WJ, Giammarile F, Tatsch K, Eschner W, et al. FDG PET/CT: EANM procedure guidelines for tumour imaging: version 2.0. Eur J Nucl Med Mol Imaging. 2015;42:328-54.

37. Working Group for Investigation and Research on Nuclear Medicine Image Quantification and Standardization JSoNMT. Point of acquisition, processing, display and output for standardized images with clinical usefulness (in Japanese). Kakuigaku Gijutsu. 2008;28:13-66.

\section{Submit your manuscript to a SpringerOpen ${ }^{\circ}$ journal and benefit from:}

- Convenient online submission

- Rigorous peer review

- Open access: articles freely available online

- High visibility within the field

- Retaining the copyright to your article

Submit your next manuscript at $>$ springeropen.com 